\title{
Listening to laser light interactions with objects of art: a novel photoacoustic approach for diagnosis and monitoring of laser cleaning interventions
}

\author{
George J. Tserevelakis ${ }^{*}$ (i), Paraskevi Pouli and Giannis Zacharakis
}

\begin{abstract}
Photoacoustic (PA) imaging is a novel, rapidly expanding diagnostic technique, which has been predominately developed in the context of contemporary biomedical research studies. In this review, we demonstrate how PA technologies can break through the barriers of biomedicine to find innovative applications in cultural heritage $(\mathrm{CH})$ diagnostics and laser cleaning monitoring. Having over three orders of magnitude higher transmission through strongly scattering media, compared to light in the visible and near infrared, PA signals offer substantially improved detection sensitivity, providing optical absorption contrast at high spatial resolution. This unique combination of features is employed for establishing novel diagnostic methodologies aiming to uncover well-hidden features and provide structural information in multi-layered $\mathrm{CH}$ objects such as paintings and documents. Finally, we demonstrate that the PA effect can be successfully utilized for the reliable monitoring of laser cleaning interventions on stonework, allowing for a safe and well-controlled cleaning procedure which will safeguard $\mathrm{CH}$ objects' original surfaces. Simplicity of implementation, effectiveness and low-cost features provided by the developed diagnostic and monitoring systems, highlight the rich potential of emerging PA technologies in $\mathrm{CH}$ studies and offer exciting possibilities for future implementations.
\end{abstract}

Keywords: Photoacoustic, Imaging, Diagnosis, Artwork, Cultural heritage, Monitoring, Laser cleaning

\section{Introduction}

The scientific investigation of cultural heritage $(\mathrm{CH})$ objects using advanced non-invasive analytical methodologies and diagnostic tools is among the first priorities of heritage scientists, conservators and restorers [1]. Several techniques which are already firmly established in other fields, such as analytical chemistry and biomedical imaging, are often utilized for the study of multifaceted $\mathrm{CH}$ assets, aiming at the extraction of valuable information related to the complex structure and composition in various items of historical significance. Undoubtedly,

*Correspondence: tserevel@iesl.forth.gr

Foundation for Research and Technology Hellas, Institute of Electronic

Structure and Laser, N. Plastira 100, 70013 Heraklion, Crete, Greece optical diagnostic techniques are considered to be among the most important tools for the investigation of $\mathrm{CH}$ objects, nevertheless, they are drastically limited to superficial observations, as a result of the intense scattering and absorption of light by several optically opaque media composing artworks (e.g. wooden or stone substrates, paints etc.). Inevitably, this fundamental limitation of pure optical methods, hinders the visualization of features and the recording of useful information from hidden layers found well-below artworks' surface. During the last decades, several optical imaging modalities have been employed for $\mathrm{CH}$ studies including near infrared (NIR) reflectance imaging, optical coherence tomography (OCT), non-linear microscopy (NLM) and THz imaging. 
More specifically, NIR Reflectance imaging has the potential to provide a mapping of hidden features in paintings, provided that the pigments are highly transparent in the NIR region and the overlying paint layers are relatively thin [2-4]. On the other hand, OCT has been shown to offer adequate spatial resolution and contrast as regards the imaging of semi-transparent media such as varnish layers, or even thin paint layers $[5,6]$. Furthermore, NLM approaches such as Multiphoton Excitation Fluorescence (MPEF) and Optical Harmonics Generation (OHG), have similarly been applied for the investigation of varnishes, thin paints, as well as, the characterization of corrosion layers in silver artefacts [7-10]. Finally, THz imaging has been used to provide enhanced penetration capabilities through opaque $\mathrm{CH}$ objects with promising results [11-13], although complex and expensive experimental equipment is generally required.

In a predominant position among the most rapidly growing imaging technologies, we find photoacoustic (PA) imaging, a novel hybrid diagnostic methodology mainly developed in the context of biomedical research studies. According to current literature, it has been numerously demonstrated that PA diagnostic systems allow for in vivo, high-resolution, multi-contrast and multi-scale imaging of biological substrates, ranging from cells and tissues, to organs and small animals. Typical preclinical applications of PA imaging include the highresolution imaging of cancer tumors [14-16], the study of metabolic disorders by mapping oxygen saturation of hemoglobin $[17,18]$, and the investigation of drug release processes [19-22]. Additionally, novel clinical diagnostic systems combining traditional ultrasonography with PA imaging have recently emerged, in an effort to provide hybrid complementary contrast which can shed light on several serious pathological conditions [23].

According to the PA effect, when light of time-variable intensity (e.g. a laser pulse) is absorbed by a material, the thermoelastic expansion of the medium will give rise to a rapid pressure change, which propagates in the form of acoustic waves into the surrounding environment. Under thermal and stress confinement conditions, the initial PA pressure rise $\left(\mathrm{p}_{0}\right)$ can be expressed as a function of the local laser optical fluence value $(\mathrm{F})$ as

$$
p_{0}=\Gamma \eta_{t h} \mu_{\alpha} F
$$

where $\Gamma$ is the dimensionless Grüneisen parameter defined as

$$
\Gamma=\beta / \kappa \rho C_{V}
$$

( $\beta$ is the thermal coefficient of volume expansion, $\kappa$ the isothermal compressibility, $\rho$ is the mass density and $C_{v}$ the specific heat capacity under constant volume), $\eta_{\text {th }}$ is the percentage of pulse energy converted into heat, while $\mu_{\alpha}$ stands for the optical absorption coefficient for the employed wavelength [24]. For imaging applications, the frequency range of the generated PA waves is generally found in the ultrasonic regime, usually varying from a few up to several tens of MHz. These waves are typically recorded using the same detection equipment (e.g. piezoelectric elements), similarly to traditional ultrasound imaging. As the amplitude of PA waves is directly proportional to the absorption coefficient of the medium for the irradiation wavelength, the technique provides excellent optical absorption contrast with high sensitivity [25]. Therefore, in the case of biomedical applications, PA imaging can provide strong signals from intrinsic biological absorbers such as hemoglobin, melanin and lipids, without the need of external contrast agents [26, 27]. The main advantage of PA approaches over pure light imaging modalities, lies on the ability to provide optical absorption contrast in turbid media, while retaining high spatiotemporal resolution. In this manner, PA signals offer substantially extended penetration depths (in the order of a few $\mathrm{mm}$ or even $\mathrm{cm}$ ) due to the fact that ultrasonic waves are attenuated up to three orders of magnitude less compared to optical radiation [28], while propagating through highly scattering media such as biological tissues. The trade-off between imaging depth and spatial resolution can be generally adjusted according to the detection bandwidth of the generated PA waves, permitting thus the investigation of various specimens at microscopic, mesoscopic or even macroscopic level. As it will be presented extensively further, PA imaging technologies have the potential to break through the barriers of biomedical research, finding innovative applications in $\mathrm{CH}$ diagnostics, such as the specific detection of hidden underdrawings, the uncovering of content in layered documents, and the stratigraphic analysis of painted artworks.

In contrast to the very recent implementations of PA diagnosis in $\mathrm{CH}$ field, PA monitoring of laser cleaning interventions has attracted the attention of heritage scientists already since the mid-90s [29]. Typically, laser ablation of materials presenting high optical absorption coefficients (e.g. carbon-containing crusts or deposits), generates high amplitude acoustic waves, while respective signals upon laser-matter interaction under weak absorption conditions (e.g. marble, parchment, wood or other common substrates) are considerably lower. As a first approach, the gradual amplitude attenuation of the laser-induced audible acoustic waves, was considered as a measure for the amount of material that has been removed, indicating also the time-point for the termination of ablation process. Such straightforward methods 
employing microphones for the recording of audible frequencies, were first applied to monitor the selective removal of black crusts from polluted limestone [30], as well as, the laser cleaning of several substrates including marble, copper and paper [31]. Later studies demonstrated the effectiveness of PA monitoring in the removal of paint films from stainless steel, glass and marble by recording ultrasound signals in the $\mathrm{MHz}$ regime [32], while others showed that, the exponential decay of generated acoustic waves can be associated with the decreasing thickness of black crust during its removal from sandstone substrates [33]. Furthermore, PA methodologies have been applied to determine the ablation thresholds of commercial graffiti sprays [34], and to monitor laserassisted paint removal from metallic surfaces, using the Fast Fourier Transform (FFT) of the recorded PA waveforms [35]. Nevertheless, it has to be mentioned that as realistic $\mathrm{CH}$ objects are highly heterogeneous in terms of optical, mechanical and geometrical properties, the reliable PA monitoring of laser cleaning interventions can be a highly challenging issue, requiring the development of improved experimental strategies and novel signal processing methodologies.

It must be emphasized that in most of the early-stage experiments discussed herein, a series of technical mockups were employed. The use of these mock-ups was mainly to prove the principle of operation as regards the use of PA modalities for diagnostic and monitoring purposes, being at the same time realistic concerning materials, dimensions, ageing conditions, weathering state etc. In this respect, our attention was to investigate whether PA detection is able to obtain signals from different materials, and to which extent, while its potential to determine the onsets for the different cleaning states was also investigated, in order to develop and optimize reliable monitoring approaches for laser cleaning interventions. These mock-ups allowed also extensive experimentation in order to set the operative parameters and circumvent a number of issues related to the employment of immersion media. In this direction, our most recent experiments were performed using air-coupled transducers to ensure that non-contact and non-invasive diagnostic and monitoring conditions can be effective. Having accomplished this mission, current experimentation is now focused on the use of real samples/objects and the monitoring of actual laser cleaning cases in order to fine-tune PA approaches, taking into account the distinctive characteristics of the actual diagnostic and cleaning challenges. The objective of this paper is to provide a brief review on the novel applications of PA diagnosis and monitoring in several challenges related to $\mathrm{CH}$, as has been recently demonstrated by research groups of IESL-FORTH (Institute of Electronic Structure and
Laser - Foundation for Research and Technology Hellas). Finally, the development of optimized, compact and low-cost instrumentation dedicated to $\mathrm{CH}$ applications is discussed, aiming to expand the capabilities of PA methodologies by overcoming current technical limitations appearing in this initial research stage.

\section{Detection of hidden underdrawings in paintings}

Underdrawings typically represent the original sketch designed by the artist to portray the theme of the painting and guide the development of the artwork. In most cases, underdrawings are generated directly on a prepared canvas using charcoal, graphite or carbon black paint. Inevitably, the subsequent covering of such preparatory drawings with several optically opaque paint layers, conceals this important part of artistic creation, hindering the assessment of the degree to which the artist followed faithfully the original sketch. Underdrawings or underpaintings have been of exceptional interest to scholars for long time [36-38], because they contain significant information as regards the working practice of a particular artist or workshop. The non-destructive detection of these hidden features may reveal the social, historical, geographical or even the psychological framework of the artistic creation, providing thus invaluable knowledge not only to art historians, but also to heritage scientists who are interested in the determination of artworks authenticity.

To face these challenges, a novel PA imaging approach has been recently introduced for the specific detection of underdrawings in paintings [39], aiming to overcome several major limitations of pure optical imaging techniques, mostly related to the intense light scattering in typical artistic materials. The prototype imaging apparatus (Fig. 1a) employs a Q-Switched Nd:YAG laser source emitting nanosecond pulses (pulse width: $10 \mathrm{ns)}$ at $1064 \mathrm{~nm}$, for the efficient excitation of PA signals. The NIR radiation is weakly focused by a low numerical aperture objective lens on the reverse side of the mockup painting sample, namely on the canvas support. The strong light scattering generates a broad illumination region, inducing the generation of strong PA signals in the highly absorbing regions of the pencil underdrawing pattern. The intensity profile of the resulting laser spot on the hidden underdrawing's plane had a $1 / \mathrm{e}^{2}$ width equal to $1.31 \mathrm{~mm}$. By considering this value as the effective diameter of the energy deposition region and by taking into account that the pulse energy was measured at $1.14 \mu \mathrm{J}$, a laser fluence value of $0.085 \mathrm{~mJ} / \mathrm{cm}^{2}$ was calculated as incident on the underdrawing's layer, assuming $100 \%$ transmission of the canvas. As the overlying oil paint layers are virtually transparent in NIR wavelengths, this imaging methodology can reveal the hidden sketch 

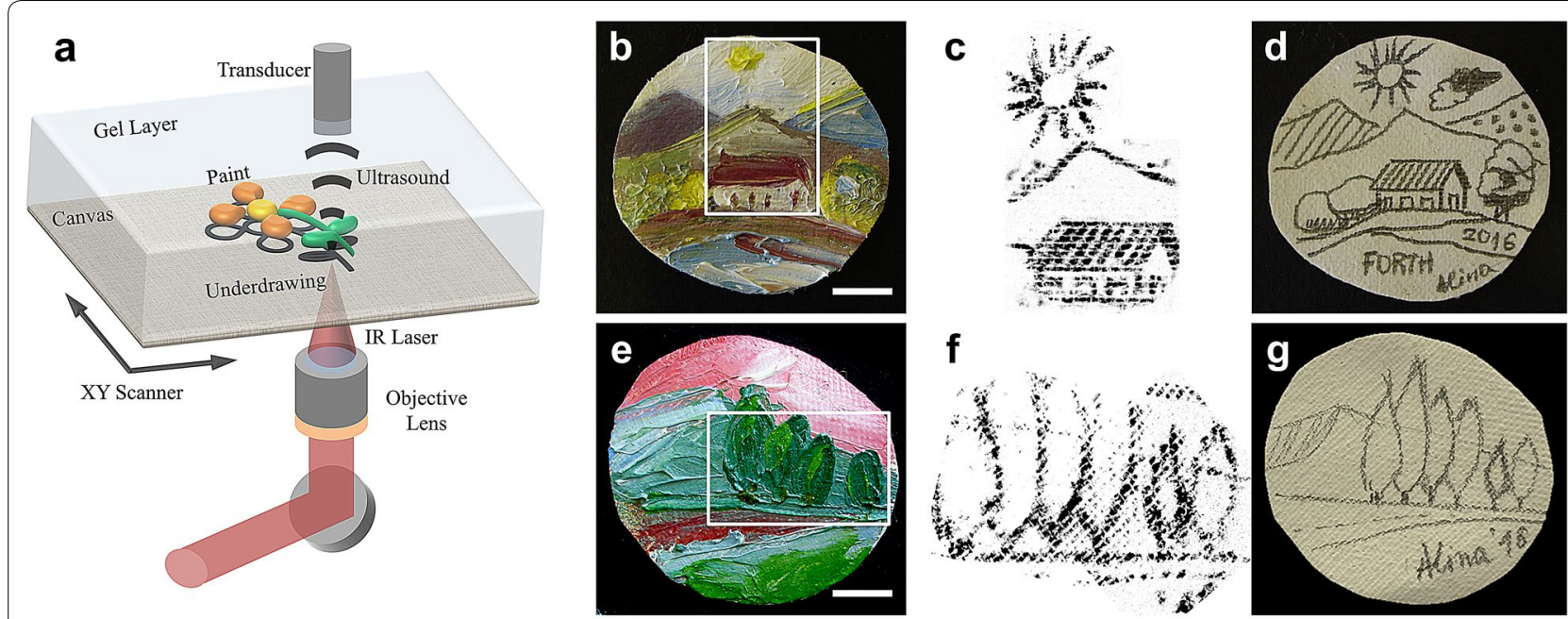

Fig. 1 PA imaging for the detection of hidden underdrawings in miniature paintings. a 3D representation of the developed experimental apparatus. b Brightfield view of miniature painting (rural landscape). c MAP PA image of the painting's underlying sketch over a central region of $2.2 \times 3.8 \mathrm{~cm}^{2}$, as indicated by the white box in (b). $\mathbf{d}$ Brightfield view of the original pencil sketch prior to overpainting. e Brightfield view of a second miniature painting (forest landscape). $\mathbf{f ~ M A P ~ P A ~ i m a g e ~ o f ~ t h e ~ u n d e r d r a w i n g ~ f o r ~ t h e ~ i n d i c a t e d ~ r e g i o n ~ i n ~ ( e ) ~ w i t h ~ l a t e r a l ~ d i m e n s i o n s ~ e q u a l ~ t o ~}$ $3.4 \times 2.4 \mathrm{~cm}^{2}$. $\mathbf{f}$ Original pencil sketch before overpainting. Both scalebars: $1 \mathrm{~cm}$. Figure adapted from [39]

with high contrast specificity. The PA signal is detected by a spherically focused ultrasonic transducer with a central frequency at $20 \mathrm{MHz}$, providing a lateral resolution in the order of $500 \mu \mathrm{m}$. The detector is immersed in a carboxymethyl cellulose (CMC) gel layer applied on the front surface of the painting and serving as an immersion medium for the effective propagation of PA waves. CMC gel is generally considered to be a compatible and safe medium for artworks, as it has been extensively used, in the form of gelling agent, for the surface cleaning of various artefacts [40-44]. The Maximum amplitude projection (MAP) PA image is generated by raster scanning the painting using a motorized $\mathrm{XY}$ stage to achieve a point by point data acquisition over the region of interest.

A brightfield image of a miniature oil painting portraying a rural landscape is shown in Fig. 1b. Pigments such as zinc white, ultramarine blue, cadmium red and chrome yellow were used for painting this miniature. A $2.2 \times 3.8 \mathrm{~cm}^{2}$ region indicated by the white box in Fig. $1 \mathrm{~b}$ was scanned using the PA imaging system, to reveal the underlying pencil drawing with high specificity (Fig. 1c). For comparison reasons, the initial pencil sketch prior to the application of oil paints is shown in Fig. 1d. To further explore the capabilities of the proposed imaging methodology, a second miniature painting was examined, portraying a forest landscape (Fig. 1e). Following the generation of an initial pencil sketch, the mock-up was painted over using a different pigment scheme including a combination of zinc white, chromium green, ultramarine blue, vermillion and azurite. In a similar manner to the previous painting mock-up, a selected region of $3.4 \times 2.4 \mathrm{~cm}^{2}$ (white box in Fig. 1e) was imaged, as presented explicitly in Fig. 1f. The acquired imaging contrast is compared to the original drawing before the application of the paint (Fig. 1g), validating the high selectivity of PA imaging in the detection of hidden pencil sketches, with minimum interference from the overlying paint layers.

\section{Uncovering the hidden content of layered documents}

Documents have existed in various forms since the fundamental invention of writing, a tool that revolutionized communication capabilities in human societies enabling, among others, the rapid exchange of information and history recording. Most of the materials that have been used for the generation of documents, such as paper, papyrus and parchment, are generally very sensitive in external conditions, often suffering from severe deterioration issues that limit their unrestricted handling for studying purposes. This puts various barriers to scholars and hinders their access for further investigations, urging for noninvasive analytical approaches that will allow access to the content of such historical objects without any further risk. In an attempt to address these challenges, a PA imaging methodology, similar to the detection of underdrawings in paintings, has been developed for the extraction of hidden content in layered documents [45]. In this case though, the irradiation wavelength was switched to $532 \mathrm{~nm}$, to increase further the 
optical absorption from carbon-based inks, enhancing thus the amplitude of the generated PA waves for the applied energy fluence. The pulse energy delivered on the document samples was less than $600 \mathrm{~nJ}$, whereas the respective focal spot at the back side of the first layer had a diameter of approximately $6 \mu \mathrm{m}$ ( $1 / \mathrm{e}^{2}$ value), yielding a maximum energy fluence at $\sim 2 \mathrm{~J} / \mathrm{cm}^{2}$. Due to the high optical scattering of paper though, the actual fluence on the first printed surface has been estimated to be smaller than $1 \mathrm{~J} / \mathrm{cm}^{2}$. Furthermore, the energy fluence is considerably lower for the rest of the layers, as a result of intense optical scattering phenomena. The technical mock-ups employed in this study were composed of several pages taken from a commercially available book, which were cut into square pieces of 5 by $5 \mathrm{~cm}^{2}$ and stacked one above the other. The thickness of the printed uncoated wood pulp paper sheets was determined using brightfield microscopy prior the PA imaging session at approximately $150 \mu \mathrm{m}$.

Time-domain PA signals were recorded from the mock-up consisting of four stacked paper sheets, single-sided printed and without any overlapping text among them. An aggregate graph of the acquired data shown in Fig. 2a, reveals explicitly the temporal separation of signals by an interval of $\sim 150 \mathrm{~ns}$, corresponding to an axial distance between the sheets equal to $225 \mu \mathrm{m}$. A gradual reduction of the maximum PA amplitude is clearly observed, by moving from the first paper sheet facing directly the laser beam, towards the fourth layer, which is found closer to the ultrasonic transducer. Such behavior can be explained in terms of a progressive local energy fluence degradation, due to the high optical scattering properties of paper sheets, as it is also described by an exponential decay fitting of the respective maximum amplitude points (Fig. 2a, dashed curve).

Three depth color-coded PA reconstructions of the four-layer document mock-up are demonstrated in Fig. 2b. The presented top (XY) and side view (XZ, YZ) MAP PA images reveal clearly the distribution of printed ink, even in the case of the fourth paper sheet, located at a relative depth of $\sim 700 \mu \mathrm{m}$. To compensate for the previously observed signal decay (Fig. 2a), the brightness of the acquired slice images has been adjusted according to the estimated fitting model. Moreover, the aspect ratio of the side view images has been purposely altered by exaggerating $\mathrm{Z}$-axis four times, enhancing thus the visual representation quality of the successive layers. The contrast specificity, spatial resolution and penetration depth demonstrated by this proof of concept study, highlight the promising potential of PA imaging methods as regards the delineation of hidden content in several types of layered documents.

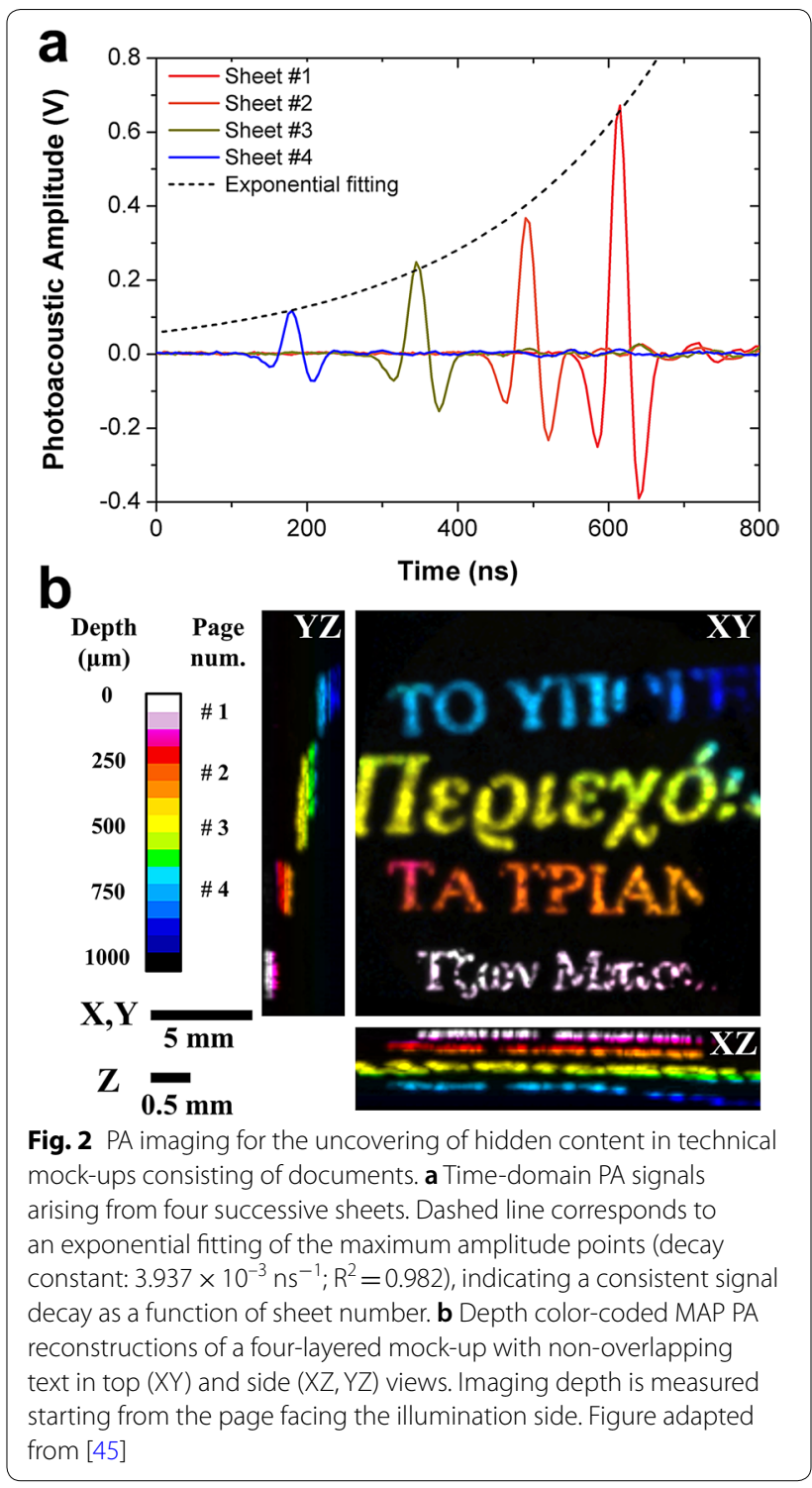

\section{Estimating the thickness of thin paint layers by means of PA signal attenuation}

The effective non-invasive stratigraphic investigation of highly complex systems such as paintings, still constitutes an open issue in $\mathrm{CH}$ diagnostics. The challenge here, lies in the fact that, the presence of several overlapping opaque layers sets limits as regards the in-depth extraction of structural and morphological features through the use of optical wavelengths. Nevertheless, the capability to detect underpaintings or underdrawings, glazes, lacquers, or even the pattern of superposed brushstrokes, may be very useful for studies related to artworks' authenticity. Moreover, the visualization of superficial or internal detachments and cracks due to ageing, becomes essential in view of consolidation 
treatments. Finally, the precise spatial mapping of aged varnishes and pictorial retouches compromising the legibility of the painted surface, could significantly benefit relevant selective cleaning interventions, promoting thus an improved restoration outcome.

Photoacoustic Signal Attenuation Analysis (PAcSAA) is a recently introduced method $[46,47]$ that could be employed to provide thickness assessment of thin optically opaque layers, typically met in paintings, with $\mu \mathrm{m}$ resolution. PAcSAA technique is based on the fact that, as generated PA waves are transmitted through lossy media, a portion of acoustic energy is gradually degraded into thermal energy due to heat diffusion and momentum exchange among the vibrating molecules [48]. The combined result of these two physical mechanisms is the attenuation of the detected PA signals according to the equation [49]

$$
p(z)=p_{0} e^{-a(f) z}
$$

where $p(z)$ is the pressure amplitude of a plane monochromatic PA wave at distance $\mathrm{z}, p_{0}$ is the initial pressure, and $a(f)$ the frequency-dependent acoustic attenuation coefficient, usually expressed in $\mathrm{dB} / \mathrm{cm}$. For many common media such as polymers or biological tissues, the latter coefficient has an approximately linear dependence on frequency, entailing a stronger attenuation effect on the higher frequency components of the broadband PA wave $[48,49]$. To investigate the PA attenuation effect, thin Primary Red Magenta paint layers of various thickness $(50,100$ and $200 \mu \mathrm{m})$ were applied on a common coverslip glass. The layers were subsequently excited by focusing a $532 \mathrm{~nm}$ beam on the back side of the sample, at the interface between the paint layer and the glass substrate. The pulse energy at the focal point ( $1 / \mathrm{e}^{2}$ width: $\left.6 \mu \mathrm{m}\right)$ was estimated to be $200 \mathrm{~nJ}$, corresponding to a maximum energy fluence in the order of $700 \mathrm{~mJ} / \mathrm{cm}^{2}$. It is worth mentioning that such fluence values are used exclusively for calibration procedures in mock-ups requiring high SNR values. The actual energy fluence in paintings is considerably lower as a result of the strong light scattering by the canvas substrate. The generated PA waves were then attenuated through the layer before being detected by a broadband spherically focused transducer (Fig. 3a). Prior the realization of PAcSAA measurements, OCT imaging was used to verify the thickness of each paint layer with high accuracy (Fig. 3b-d). The detected time-domain PA signals for the three examined layers were shifted into the frequency domain through a FFT operation, to generate the respective normalized PA amplitude spectrum for each case (Fig. $3 \mathrm{e}-\mathrm{g}$ ). It was observed that the spectra were composed of two distinct frequency bands; a lower one between $10-80 \mathrm{MHz}$, and a higher one ranging from
80-140 MHz. Dashed curves in Fig. 3e-g demonstrate a $4^{\text {th }}$ order polynomial fitting for the lower frequency bands, indicating a PA red-shift with an increasing layer thickness, as it is clear from the estimated maximum amplitude frequency values $(43.76 \mathrm{MHz}$ for the $50 \mu \mathrm{m}$ layer, $41.44 \mathrm{MHz}$ for the $100 \mu \mathrm{m}$ layer and $35.37 \mathrm{MHz}$ for the respective $200 \mu \mathrm{m}$ layer). To provide a measure of the overall PA attenuation, the Average Transmitted Frequency (ATF) was calculated for an effective detection band of $10-130 \mathrm{MHz}$ according to the formula

$$
A T F=\sum_{f_{i}=10}^{130 \mathrm{MHz}} A\left(f_{i}\right) f_{i} / \sum_{f_{i}=10}^{130 \mathrm{MHz}} A\left(f_{i}\right)
$$

where $A\left(f_{i}\right)$ is the normalized amplitude of each frequency component $f_{i}$. A plot of ATF values as a function of the OCT measured layer thickness is presented in Fig. 3h. Black curve corresponds to the fitting of experimental points by an exponential decay function, whereas error bars represent the standard deviation on five sequential ATF measurements in different areas of the sample. The fitted curve was subsequently used for the investigation of a realistic canvas painting mock-up, composed of a pencil underdrawing and an overlying Primary Red Magenta paint layer. Irradiation at $1064 \mathrm{~nm}$ revealed the hidden sketch with high fidelity (Fig. 3i) due to the strong absorption of graphite, while by shifting into the $532 \mathrm{~nm}$ wavelength, a rather uniform MAP PA image was recorded (Fig. 3j) as a result of the significant absorption by the overlying paint. By applying PAcSAA method to the recorded waveforms, a paint layer thickness map could be extracted (Fig. 3k) according to the previously estimated model correlating ATF values with OCT measurements. These results provide experimental evidence that PA excitation through the fundamental and second harmonic wavelength of commonly available Nd:YAG laser sources, can provide both cross-sectional information of painted layers and delineation of features hidden underneath the paints with high accuracy and contrast specificity.

\section{Combined PA and NLM imaging for stratigraphic analysis of paintings}

During the last decade, NLM approaches have been introduced in the context of $\mathrm{CH}$ diagnostics in an effort to extend the maximum imaging depth into scattering media, by taking advantage of the higher transmissivity of NIR wavelengths. In this direction, several studies have demonstrated that non-linear contrast modes such as Multiphoton Excitation Fluorescence (MPEF), Second Harmonic Generation (SHG) and Third Harmonic Generation (THG), can be successfully applied to the indepth imaging of fresh and aged varnishes, lining glues, historical coatings, parchments, paints and corrosion 

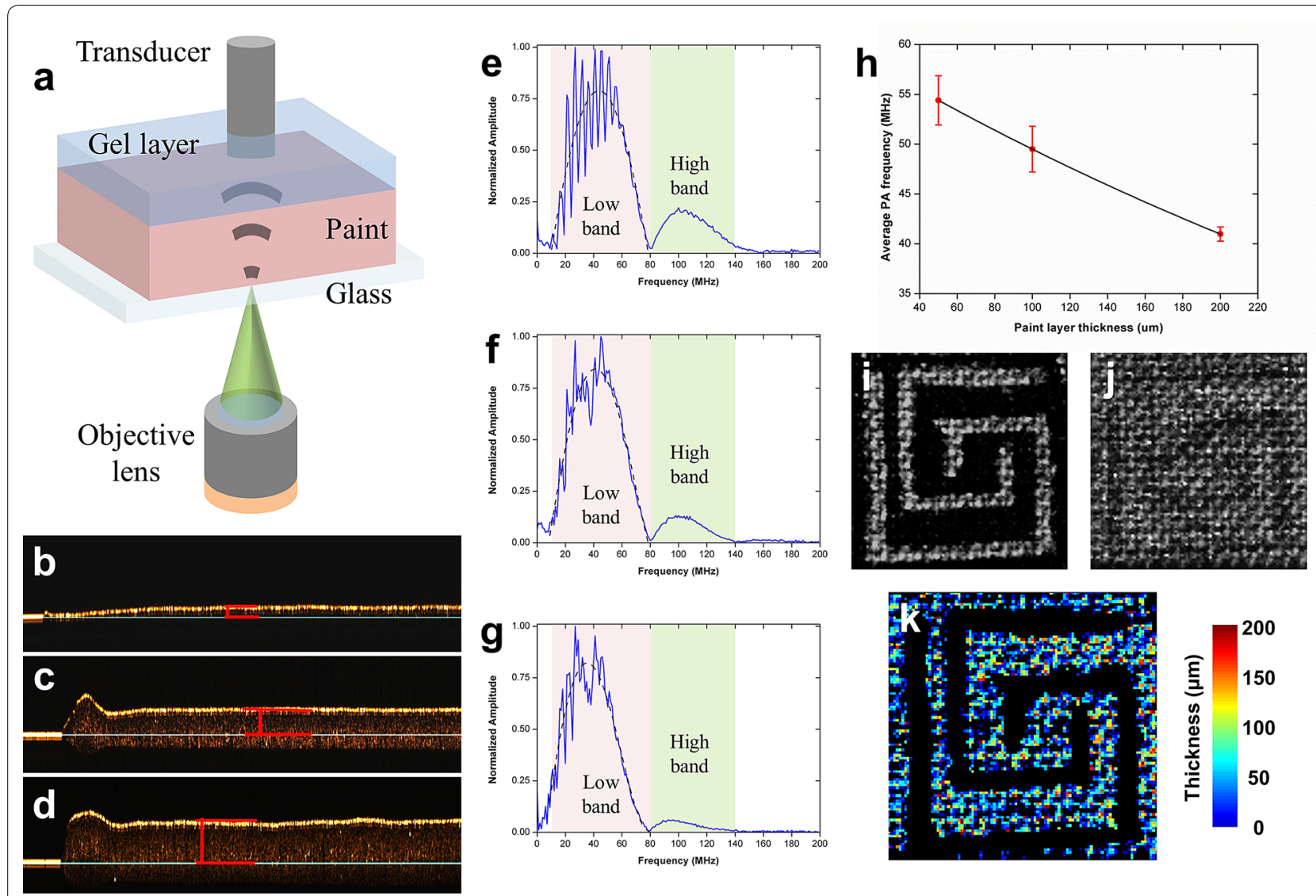

Fig. 3 PACSAA method for the assessment of thin layers thickness in paintings. a PACSAA experimental setup. b-d OCT profiles of three primary red magenta paint layers on glass substrates, with measured thickness (red bars) at 50, 100 and $200 \mu \mathrm{m}$ respectively. e-g Corresponding normalized PA amplitude spectra for the primary red magenta paint layers presented in (b-d). Dashed curves indicate a 4th order polynomial fitting for the predominant low-frequency bands (10-80 MHz). $\mathbf{h}$ Plot of ATF versus OCT measured thickness for the investigated paint layers. Error bars correspond to the standard deviation of 5 sequential ATF measurements performed at different regions of the mock-up. Black line represents the fitting of the experimental points using an exponential decay function. i MAP PA image of a painting mock-up at $1064 \mathrm{~nm}$. $\mathbf{j}$ MAP PA image of the same mock-up using the second harmonic wavelength at $532 \mathrm{~nm}$. $\mathbf{k}$ PAcSAl image of color-coded paint thickness values estimated according to the exponentially fitted curve shown in (h). Figure adapted from [46] and [47]

layers in metal artefacts [7-10, 50-54]. Nevertheless, several materials typically met in works of art are optically opaque to such a degree that they do not permit observations beyond a few $\mu \mathrm{m}$ in depth using exclusively optical radiation. To provide an augmented diagnostic approach offering multi-contrast information together with a high penetration capability in opaque media, a combination of PA imaging and NLM has been recently demonstrated [55], with emphasis given on the stratigraphic analysis of painted artworks.

Figure 4.1a shows a cross-sectional MPEF image (excitation at $1028 \mathrm{~nm}$ ) of a canvas painting mock-up, for a $\mathrm{XZ}$ region of $1 \mathrm{~cm}$ by $385 \mu \mathrm{m}$ sampled with 200 by 80 pixels respectively. By applying a custom-made deconvolution-based algorithm, the mixed information carried by the MPEF image was decomposed into relative contributions from the overlying mastic layer (Fig. 4.1b) and the applied red lead paint (Fig. 4.1c), presented with blue and red colors respectively. The cross-sectional PA image from the same region (excitation at $1064 \mathrm{~nm}$ ) shown in Fig. 4.1d with green color, reveals explicitly hidden pencil underdrawings for the selected plane. A composite RGB image merging the sections presented in Fig. $4.1 \mathrm{~b}, 1 \mathrm{c}$ and $1 \mathrm{~d}$ is shown in Fig. 4.1e, delineating the full stratigraphy of the investigated mock-up within the performance limits of the combined imaging modes (i.e. the deeper part of paint layer cannot be imaged due to high optical scattering and absorption). To validate the extracted data, the composite image has been subsequently compared with cross-sectional brightfield microscopy imaging, after the painting sample is cut. Figure 4.1f shows a near-UV brightfield view of the mock-up's cross section at the region indicated with a white square in Fig. 4.1e. The images appeared to present a high degree of similarity in 

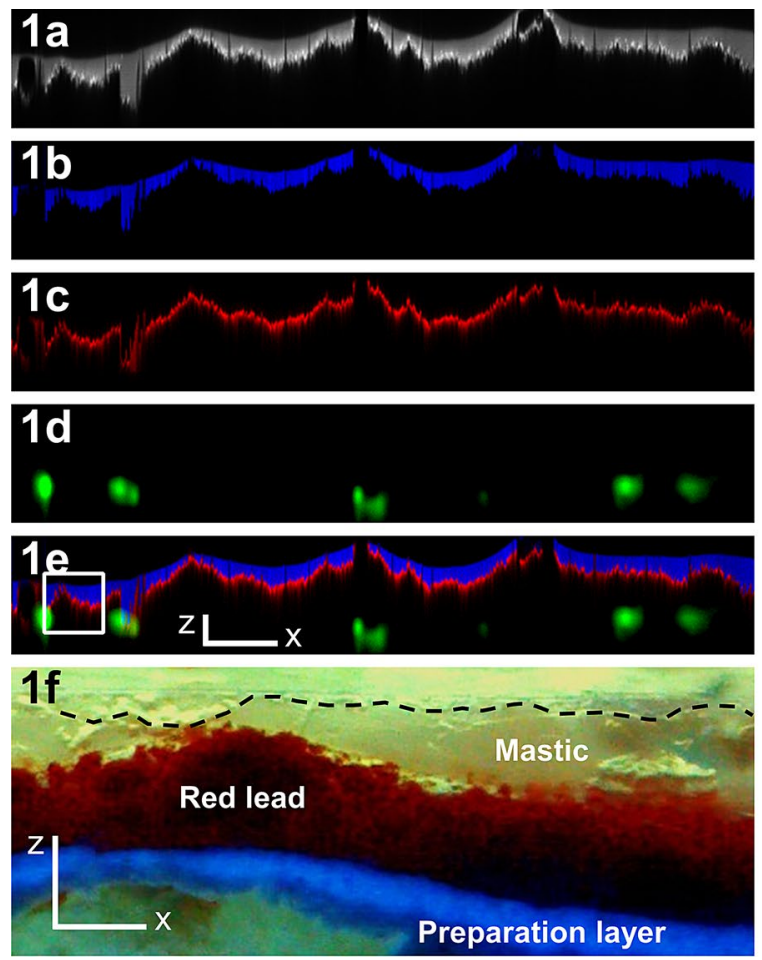

Fig. 4 Combined NLM and PA stratigraphic imaging and AC PA detection of hidden underdrawings in painting mock-ups. 1a Raw cross-sectional MPEF image of the mock-up. 1b Unmixed MPEF image of the overlying mastic layer. 1c Unmixed MPEF image of red lead paint layer. 1d PA image of underlying pencil sketch. 1e Composite MPEF and PA image (Scalebars Z: $100 \mu \mathrm{m} ; \mathrm{X}: 1 \mathrm{~mm})$. 1f Brightfield image of the region indicated with a white square in (1e) using near-UV light. The black dashed line represents the border of mastic layer. Both scalebars in Z and X dimensions correspond to $100 \mu \mathrm{m}$. 2a Brightfield view of a painting mock-up using an ultramarine blue paint layer. $\mathbf{2} \mathbf{b}$ Brightfield view of the underlying pencil sketch prior overpainting. 2c Recovered pencil underdrawing pattern through AC PA imaging. 2d NIR optical image of the painting sample recorded at $1050 \mathrm{~nm}$. Similar data are presented for chromium green ( $\mathbf{e} \mathbf{e}-\mathbf{2} \mathbf{h})$, vermillion ( $\mathbf{2} \mathbf{i}-\mathbf{2} \mathbf{l})$ and zinc white ( $\mathbf{2 m}-\mathbf{2} \mathbf{p})$ paints. All scalebars are equal to $5 \mathrm{~mm}$. Figure adapted from [55] and [56]

terms of structure, morphology and layers' spatial distribution on the canvas. Furthermore, the extracted average mastic layer thickness value for the right section of the region of interest $(\sim 75 \mu \mathrm{m})$ was found to be in very good agreement with Fig. 4.1f, confirming thus the accuracy of the adopted imaging approach.

\section{Air-coupled PA detection of hidden underdrawings in paintings}

The high signal transmissivity and the optical absorption contrast specificity provided by PA imaging, constitute the most important features allowing for effective in-depth investigations of several optically opaque $\mathrm{CH}$ objects. Nevertheless, the sensitive detection of PA signals typically requires the use of immersion ultrasonic transducers and respective compatible coupling media in direct contact with artwork's surface. Despite the fact that standard gelling agents such as CMC, are commonly used for restoration operations as safe and highly compatible media [40-44], it has to be considered that several sensitive surfaces of an artwork would not tolerate, in any case, even a minimum contact with some gel material. To overcome this issue and advance PA technology towards fully non-contact and non-invasive $\mathrm{CH}$ diagnostic approaches, an optimized imaging system integrating a spherically focused air-coupled (AC) ultrasonic transducer (central frequency: $1 \mathrm{MHz}$ ) has been developed [56]. In this case, a collimated pulsed NIR laser beam has been employed for the efficient excitation of PA signals (pulse energy: $4.5 \mathrm{~mJ}$; maximum energy fluence: $\left.140 \mathrm{~mJ} / \mathrm{cm}^{2}\right)$. To demonstrate the capabilities of the developed system for the uncovering of hidden underdrawings, painting samples were prepared with the aim to replicate real easel paintings in a small scale. Figure 4.2a shows the brightfield image of a sample overpainted with an ultramarine blue layer, whereas Fig. 4.2b depicts the underlying pencil sketch before the application of the paint. Figure $4.2 \mathrm{c}$ corresponds to a MAP PA image of the pencil underdrawing, as a result of the selective absorption of infrared radiation $(\lambda=1064 \mathrm{~nm})$ by the graphite deposition regions. The AC PA diagnostic approach was further compared with NIR imaging 
(Fig. 4.2d) within a similar optical spectral band (central wavelength: $1050 \mathrm{~nm}$, FWHM: $25 \mathrm{~nm}$ ). As it is clear from the respective figures, the two imaging methods provide highly comparable results for the case of ultramarine blue paint in terms of spatial resolution and contrast specificity. Similar data are presented for a chromium green painting sample (Fig. $4.2 \mathrm{e}-\mathrm{h}$ ), validating the capabilities of AC PA diagnosis as regards the accurate detection of underdrawings. Figure $4.2 \mathrm{i}$ depicts a painting sample covered with vermillion red paint, whereas Fig. 4.2j shows the pencil sketch prior overpainting. As it is clear from Fig. 4.2k, PA imaging has been able to recover successfully the hidden sketch, with reduced contrast though, due to the strong optical absorption background of the overlying paint. In contrast to these results, the respective NIR image (Fig. 4.2l) was not able to provide any information as regards the underlying pencil sketch. A similar relative performance was also observed in the case of a zinc white paint sample (Fig. $4.2 \mathrm{~m}$ ), presenting high reflectivity and optical scattering properties. The hidden pencil sketch (Fig. 4.2n) was reconstructed with satisfactory contrast levels using AC PA imaging (Fig. 4.2o) due to the negligible optical absorption background. On the contrary, the respective NIR image shown in Fig. 4.2p, was unsuccessful to provide useful contrast of the pencil pattern, due to the extreme light reflection and diffusion in the paint layer. While the presented system is developed in a trans-illumination configuration, which limits its application to relatively thin artworks only, an epi-illumination geometry integrating laser excitation and $\mathrm{AC}$ PA detection on the same side could be adopted to offer universal diagnostic capabilities for artworks of arbitrary thickness and shape.

\section{A statistical approach for the on-line PA monitoring of laser cleaning on stone}

Laser-assisted removal of unwanted layers is wellestablished in $\mathrm{CH}$, however material extraction and potential side-effects to the substrate must be reliably determined and monitored. Several analytical techniques have been employed after cleaning [57-59], but on-line assessment of the process itself and its consequences on the authentic surface is still an open issue. In this context, a novel statistical methodology has been recently introduced for the reliable monitoring of laser cleaning on stone, integrating low noise PA amplitude measurements in the $\mathrm{MHz}$ regime [60]. The method aims at establishing correlations between PA signal levels and the onsets of over-layer ablation, effective cleaning and substrate's damage stages during laser cleaning interventions. For this reason, a simple and straightforward case application has been considered; namely the removal of black graffiti paint from medium-coarse grained white marble. A schematic representation of the PA monitoring setup is shown in Fig. 5a. A contact piezoelectric transducer with central frequency at $5 \mathrm{MHz}$ detected the generated PA waves during the irradiation of the paint layer with the fundamental wavelength of a Nd:YAG laser. The amplified signals were recorded by an oscilloscope and processed using the modulus of Hilbert transformation, to estimate an average amplitude value within a selected temporal window of $500 \mu$ s following the pulse incidence. Figure 5b shows Scanning Electron Microscopy (SEM) images of representative laser spots at the onset of paint ablation, effective cleaning, and substrate damage stages, for different irradiation fluence values (0.6, $1.0,1.5$ and $2.0 \mathrm{~J} / \mathrm{cm}^{2}$ respectively). The surface roughness for the damage stage, estimated using confocal microscopy, presented a significant and abrupt increase in comparison to the typical roughness recorded at the effective cleaning stage (top right values of images in Fig. 5b). Such an increase was consistent even in cases without evident structural changes in the respective SEM images. Subsequently, these combined observations were correlated with the respective PA signals generated on the last pulse incidence, in an attempt to predict the three onsets for a selected energy fluence value. Linear regression models presented explicitly in Fig. $5 \mathrm{c}$, were generated to demonstrate the strong linear relation between the investigated variables for the cleaning and damage onsets $\left(R^{2}>0.98\right)$, as well as, the approximately fluence-independent behavior of the normalized average PA signal regarding the paint ablation onset.

Finally, color-coded graphs of the normalized PA signal evolution were generated as a function of the number of applied pulses. The different colors represented the extent of ablation (gray), cleaning (green) and damage (red) stages, as predicted by the fitted linear regression models. Figure $5 \mathrm{~d}-\mathrm{g}$ show the color-coded PA signal evolution during the incidence of fifteen sequential pulses at four applied fluences. The three stages were delimited according to the intersection of each model's prediction (dashed lines in black, green and red respectively) with the original normalized PA signal curve. The presented graphs provide a visual representation of the monitoring process, revealing a progressively lower degree of control for increasing fluence values, as the effective cleaning extent appears to become narrower. Nevertheless, the consistency of the generated PA signals indicated that the models have the potential to monitor and guide the laser cleaning procedure with minimized collateral effects, even in the case of higher irradiation fluences. 


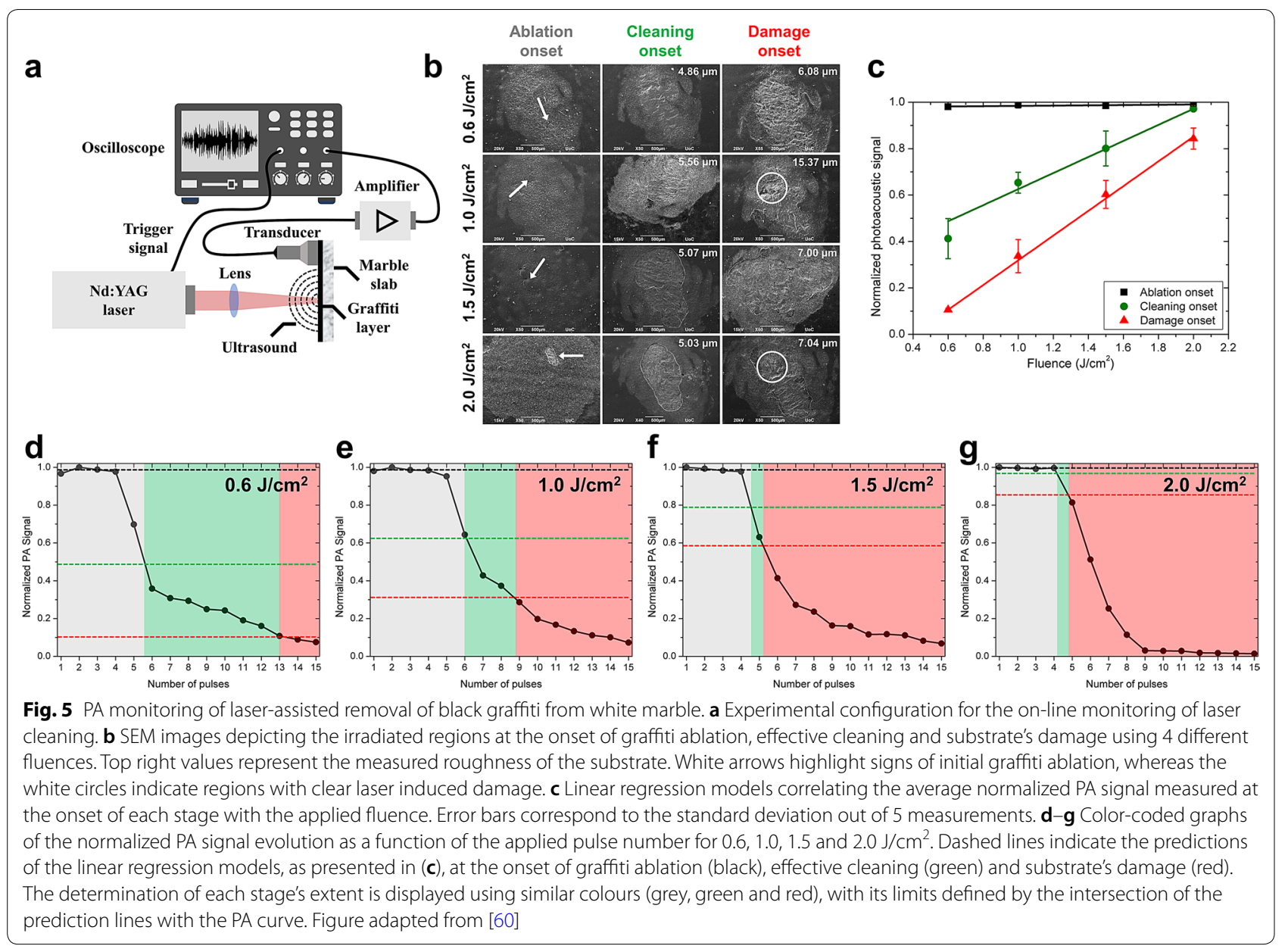

\section{Hybrid PA and optical monitoring of laser-assisted encrustation removal}

The laser-assisted removal of dark pollution crusts from stone substrates is one of the first cleaning challenges that have been effectively faced in the laser cleaning research [30]. Such crusts are often developed on the surface of monuments exposed to urban environment due to the microclimate, acid rain, humidity and airborne particles. A novel hybrid PA and optical imaging setup has been lately applied for the in-situ and real-time monitoring of laser cleaning tests, which were performed on marble mock-up samples [61]. The mock-ups simulated simplified cases of environmental encrustations, and consisted of a mixture of gypsum with $5 \% \mathrm{w} / \mathrm{w}$ carbon, in the form of charcoal powder, applied on marble plates. The hybrid setup integrated a contact transducer and a high-resolution camera, for the simultaneous recording of PA waveforms and optical images, following the incidence of NIR pulses aiming to remove the encrustation layer. A novel processing protocol of the PA waveforms was established for the detection of the laser pulse that removes the crust, revealing the marble substrate. In this context, the original PA waveform generated by the first pulse, was successively cross-correlated with the respective waveforms recorded following the incidence of all pulses (Fig. 6a). Furthermore, the maximum amplitude of the calculated cross-correlations was extracted as a function of the number of applied pulses (Fig. 6b). While these data cannot clearly denote the critical timepoint of crust removal, it was observed that the percentage change of the maximum amplitudes was capable of providing a well-distinguishable peak, indicating the $5^{\text {th }}$ pulse as the one which eliminates the unwanted material (Fig. 6c). This finding was in agreement with the synchronized optical image recording, depicting the irradiated region for each pulse incidence (Fig. 6d).

Following the developed PA signal analysis protocol, three different regimes were investigated, namely: a) damage-inducing irradiation conditions b) efficient removal of crust without damage on the substrate, and c) inefficient encrustation removal. These regimes can be observed in the graph of Fig. 6e, by applying energy 


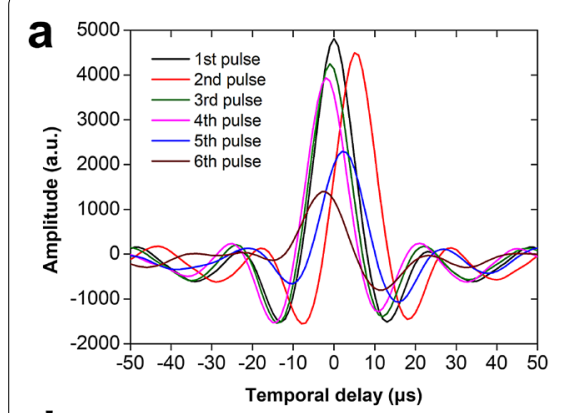

d

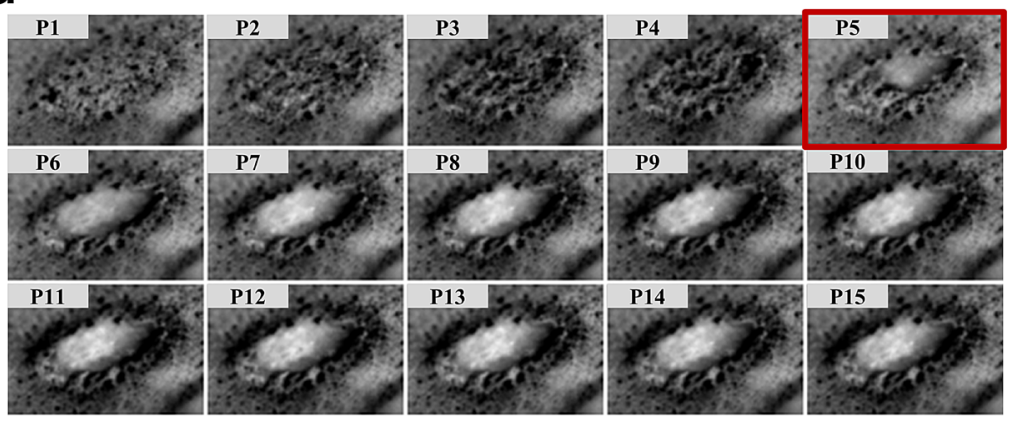

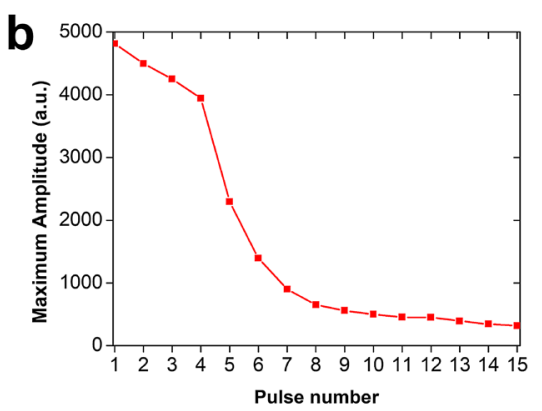
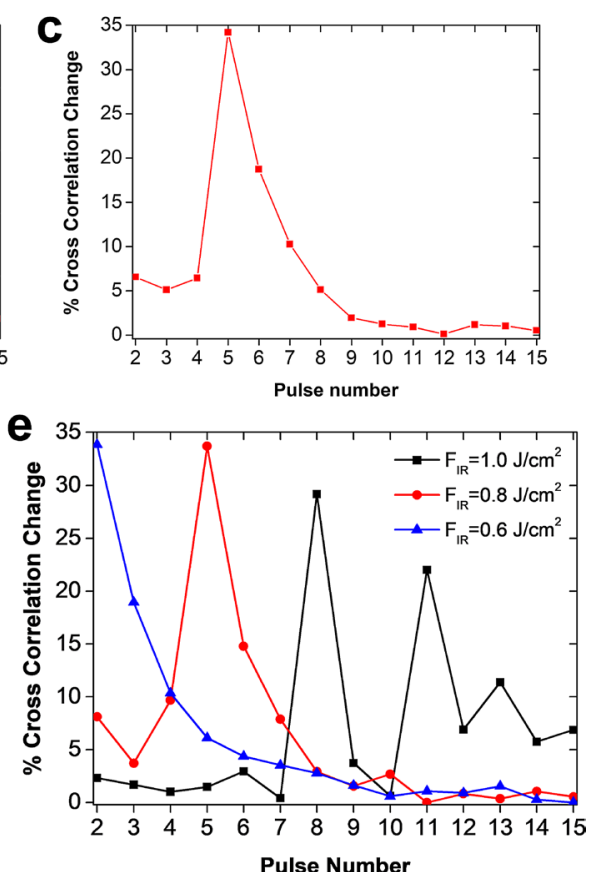

Fig. 6 Hybrid PA and optical monitoring during the laser-assisted removal of encrustation from stonework. a Cross-correlation between PA waveforms recorded after the incidence of Nth and 1st pulse, which is used as reference (first 6 pulses are presented). The 1st pulse curve (black color) corresponds to an autocorrelation function. An optimum laser fluence is applied at $0.8 \mathrm{~J} / \mathrm{cm}^{2}$. b Maximum amplitude of cross-correlation function graph estimated for 15 pulses. c Percentage change of cross-correlation maximum amplitude; the peak indicates the pulse for which the marble substrate has been reached. $\mathbf{d}$ Optical images of the laser spot recorded for 15 sequential pulses. Red border indicates the point (5th pulse) where the maximum change has been observed according to (c). e Percentage change of cross-correlation maximum amplitude estimated for 15 pulses at 3 different fluences. Figure adapted from [61]

fluence values of $1.0 \mathrm{~J} / \mathrm{cm}^{2}$ (overcleaning), $0.8 \mathrm{~J} / \mathrm{cm}^{2}$ (optimum cleaning), and $0.6 \mathrm{~J} / \mathrm{cm}^{2}$ (undercleaning) respectively. At $1.0 \mathrm{~J} / \mathrm{cm}^{2}$ (Fig. 6e; black line), PA signals reveal that the crust is removed at the incidence of the 8th pulse, whereas the additional peaks following the main one, correspond to further extraction of material, damaging the substrate. The irradiation at $0.8 \mathrm{~J} / \mathrm{cm}^{2}$ (Fig. 6e; red line) is considered to be the optimum irradiation condition, for which the deposition layer is effectively removed at the $5^{\text {th }}$ pulse, without any subsequent damage, as it was additionally verified by optical imaging and profilometry measurements. Finally, the low-fluence irradiation case (Fig. 6e; blue line) presented a total absence of peaks, indicating an ineffective cleaning of the crust which was also confirmed by optical observations.

\section{Conclusions}

In this review, we have demonstrated the potential of novel PA technologies in the diagnostics of $\mathrm{CH}$ objects, as well as, the monitoring of relevant laser cleaning interventions. The excellent optical absorption contrast features of PA imaging have permitted in-depth investigations of optically opaque $\mathrm{CH}$ mock-ups such as miniature paintings and multilayered documents. Nevertheless, the majority of these studies involves the use of immersion ultrasonic transducers and a respective coupling medium in direct contact with artwork's surface for the efficient detection of PA signals. In order to compete existing diagnostic methods commonly used in $\mathrm{CH}$ applications such as infrared imaging, OCT or X-rays, the PA technique has to be implemented in a totally noncontact and non-invasive manner. In this direction, the option of immersion medium-free PA imaging can be considered in an optimized setup integrating all-optical ultrasound detection configurations, such as interferometers, fiber-based optics approaches, waveguides, and micro-ring resonators [62]. An alternative PA detection scheme, which has already shown promising capabilities both in $\mathrm{CH}$ diagnostics [56] and biomedical imaging [63], involves the integration of highly sensitive air-coupled transducers (e.g. unfocused, spherically / cylindrically focused) with adjusted bandwidth according to the application's requirements. Such ultrasound detection approaches could also be used for the monitoring of laser cleaning interventions, avoiding thus the contact of the transducer with the object. 
Another future perspective of PA diagnosis in $\mathrm{CH}$ is the development of low-cost, compact systems integrating cheap excitation sources with high wavelength availability ranging from near UV to mid-IR, such as Light Emitting Diodes (LEDs) and diode lasers. These light sources could be directly modulated using digital or analog intensity modulation schemes to produce light pulses in the order of ns [64], or variable intensities with frequencies found in the $\mathrm{MHz}$ regime [65]. The intensity-modulation of low-cost sources could essentially overcome wavelength limitations of commonly used Q-Switched lasers, enabling multispectral PA imaging capabilities and potential differentiation of optical absorbers through specialized spectral unmixing algorithmic procedures [66].

Finally, the collection of large PA datasets in several applications, allows for the reliable training of deep neural networks and sophisticated machine learning algorithms $[67,68]$. The processing of unique PA information using these novel data analysis protocols, may highlight specific signal patterns for classification, discrimination or reconstruction artifact removal purposes, upgrading substantially the diagnostic and monitoring capabilities of the method. Within this technological framework, the development of effective, compact, low-cost and fully non-invasive PA diagnostic and monitoring systems, could attract further the interest of $\mathrm{CH}$ community, paving the way for more relevant contributions in this recently emerging research field.

\begin{abstract}
Abbreviations
PA: Photoacoustic; CH: Cultural heritage; NIR: Near infrared; OCT: Optical coherence tomography; NLM: Non-linear microscopy; MPEF: Multiphoton excitation fluorescence; OHG: Optical harmonics generation; FFT: Fast Fourier transform; CMC: CarboxyMethyl cellulose; MAP: Maximum amplitude projection; PACSAA: Photoacoustic signal attenuation analysis; ATF: Average transmitted frequency; SHG: Second harmonic generation; THG: Third harmonic generation; UV: Ultraviolet; AC: Air-coupled; FWHM: Full width at half maximum; SEM: Scanning electron microscopy; LED: Light emitting diode; SNR: Signal to noise ratio.

\section{Acknowledgements}

The Authors would like to thank all the colleagues that have contributed to the experiments towards the development of these techniques, as shown in the original publications.
\end{abstract}

\section{Authors' contributions}

All authors contributed in literature search, writing of the manuscript and final proof reading before submission.

\section{Funding}

This research was supported by the national projects a) "HELLAS CH" (MIS 5002735), b) "BIOIMAGING-GR" (MIS 5002755) both implemented under the "Action for Strengthening Research and Innovation Infrastructures" and c) "POLITEIA II" (MIS 5002478) which was implemented under the "Action for the Strategic Development on the Research and Technological Sector". All three national projects are funded by the Operational Programme "Competitiveness, Entrepreneurship and Innovation" (NSRF 2014-2020) and co-financed by Greece and the European Union (European Regional Development Fund). Furthermore, the authors would like to acknowledge support from EU H2O2O projects IPERION-CH (GA 654028) and Laserlab Europe (GA 654148). G.J.T acknowledges the financial support from the Stavros Niarchos Foundation within the framework of the project ARCHERS ("Advancing Young Researchers' Human Capital in Cutting Edge Technologies in the Preservation of Cultural Heritage and the Tackling of Societal Challenges").

\section{Data availability}

The datasets used and/or analysed during the current study are available from the corresponding author on reasonable request.

\section{Competing interests}

The authors declare that they have no competing interests.

Received: 22 June 2020 Accepted: 22 September 2020

Published online: 02 October 2020

\section{References}

1. Fotakis C, Anglos D, Zafiropulos V, Georgiou S, Tornari V. Lasers in the preservation of cultural heritage: principles and applications. New York: CRC Press; 2006.

2. Daffara C, Pampaloni E, Pezzati L, Barucci M, Fontana R. Scanning multispectral IR reflectography SMIRR: an advanced tool for art diagnostics. Acc Chem Res. 2010;43:6.

3. Liang H. Advances in multispectral and hyperspectral imaging for archaeology and art conservation. Appl Phys A Mater Sci Process. 2012;106:2.

4. Cucci C, Delaney JK, Picollo M. Reflectance hyperspectral imaging for investigation of works of art: old master paintings and illuminated manuscripts. Acc Chem Res. 2016:49:10.

5. Liang $\mathrm{H}$, et al. En-face optical coherence tomography - A novel application of non-invasive imaging to art conservation. Opt Express. 2005;13:16.

6. Targowski P, Iwanicka M. Optical coherence tomography: Its role in the non-invasive structural examination and conservation of cultural heritage objects—a review. Appl Phys A Mater Sci Process. 2012;106:2.

7. Filippidis G, Tserevelakis GJ, Selimis A, Fotakis C. Nonlinear imaging techniques as non-destructive, high-resolution diagnostic tools for cultural heritage studies. Appl Phys A Mater Sci Process. 2014;118:2.

8. Faraldi $F$, et al. Multi photon excitation fluorescence imaging microscopy for the precise characterization of corrosion layers in silver-based artifacts. Appl Phys A Mater Sci Process. 2013;111:1.

9. Selimis A, et al. Nonlinear microscopy techniques for assessing the UV laser polymer interactions. Opt Express. 2012;20:4.

10. Vounisiou $P$, et al. The use of model probes for assessing in depth modifications induced during laser cleaning of modern paintings. Appl Phys A Mater Sci Process. 2010;100:3.

11. Adam AJL, Planken PCM, Meloni S, Dik J. TeraHertz imaging of hidden paint layers on canvas. Opt Express. 2009;17:5.

12. Filippidis $\mathrm{G}$, et al. Nonlinear imaging and $\mathrm{THz}$ diagnostic tools in the service of cultural heritage. Appl Phys A Mater Sci Process. 2012;106:2.

13. Redo-Sanchez A, et al. Terahertz time-gated spectral imaging for content extraction through layered structures. Nat Commun. 2016;7:12665.

14. Manohar S, et al. Initial results of in vivo non-invasive cancer imaging in the human breast using near-infrared photoacoustics. Opt Express. 2007;15:19.

15. Mallidi S, et al. Multiwavelength photoacoustic imaging and plasmon resonance coupling of gold nanoparticles for selective detection of cancer. Nano Lett. 2009;9:8.

16. Taruttis A, Ntziachristos V. Advances in real-time multispectral optoacoustic imaging and its applications. Nat Photonics. 2015;9:4.

17. Wang LV, Hu S. Photoacoustic tomography: In vivo imaging from organelles to organs. Science. 2012;335:6075.

18. Wang X, Xie X, Ku G, Wang LV, Stoica G. Noninvasive imaging of hemoglobin concentration and oxygenation in the rat brain using high-resolution photoacoustic tomography. J Biomed Optics. 2006;11:2.

19. Kim S, Chen Y-S, Luke GP, Emelianov SY. In vivo three-dimensional spectroscopic photoacoustic imaging for monitoring nanoparticle delivery. Biomed Optics Express. 2011;2:9.

20. Lee $\mathrm{HJ}$, et al. In vitro and in vivo mapping of drug release after laser ablation thermal therapy with doxorubicin-loaded hollow gold nanoshells using fluorescence and photoacoustic imaging. J Control Release. 2013;172:1 
21. Nie L, et al. Early-stage imaging of nanocarrier-enhanced chemotherapy response in living subjects by scalable photoacoustic microscopy. ACS Nano. 2014;8:12.

22. Nie L, Chen X. Structural and functional photoacoustic molecular tomography aided by emerging contrast agents. Chemical Society Reviews. 2014;43:20.

23. Steinberg l, et al. Photoacoustic clinical imaging. Photoacoustics. 2019;14:77-98.

24. Wang LV, Wu H-I. Biomedical optics: principles and imaging. Hoboken: Wiley; 2007.

25. Yao J, Wang LV. Sensitivity of photoacoustic microscopy. Photoacoustics. 2014;2:2.

26. Yao J, Wang LV. Photoacoustic microscopy. Laser Photonics Rev. 2013;7:5.

27. Cao Y, et al. Fast assessment of lipid content in arteries in vivo by intravascular photoacoustic tomography. Sci Rep. 2018;8:1

28. Hui J, et al. Bond-selective photoacoustic imaging by converting molecular vibration into acoustic waves. Photoacoustics. 2016;4:1.

29. Cooper M. Laser Cleaning in Conservation: An Introduction. Woburn: Butterworth Heinemann; 1998.

30. Cooper MI, Emmony DC, Larson J. Characterization of laser cleaning of limestone. Opt Laser Technol. 1995;27:1.

31. Lee JM, Watkins KG. In-process monitoring techniques for laser cleaning Opt Lasers Eng. 2000;34:4.

32. Bregar VB, Možina J. Optoacoustic analysis of the laser-cleaning process. Appl Surf Sci. 2002;185:3-4.

33. Jankowska M, Śliwiński G. Acoustic monitoring for the laser cleaning of sandstone. J Cult Herit. 2003:4:333-40.

34. Gómez C, Costela A, García-Moreno I, Sastre R. Comparative study between IR and UV laser radiation applied to the removal of graffitis on urban buildings. Appl Surf Sci. 2006;252:8.

35. Villarreal-Villela AE, Cabrera LP. Monitoring the Laser Ablation Process of Paint Layers by PILA Technique. Open J Appl Sci. 2016;6:626-35.

36. Alfeld $\mathrm{M}$, et al. Visualizing the 17 th century underpainting in Portrait of an Old Man by Rembrandt van Rijn using synchrotron-based scanning macro-XRF. Appl Phys A Mater Sci Process. 2013;111:1.

37. Gavrilov D, Maeva E, Grube O, Vodyanoy I, Maev R. Experimental comparative study of the applicability of infrared techniques for non-destructive evaluation of paintings. J Am Inst Conserv. 2013;52:1.

38. Cascone, S. Hidden Portrait Discovered Beneath the Surface of Picasso Painting. Artnet News. 2014. https://news.artnet.com/exhibitions/hidde n-portrait-discovered-beneath-the-surface-of-picasso-painting-41747. Accessed 22 Jun 2020.

39. Tserevelakis GJ, et al. Photoacoustic imaging reveals hidden underdrawings in paintings. Sci Rep. 2017;7:1.

40. Feller RL, Wilt MH. Evaluation of cellulose ethers for conservation. Marina del Rey: Getty Conservation Institute; 1990.

41. Kleiner FS. Gardner's art through the ages: the western perspective. 13th ed. Boston: Wadsworth; 2010.

42. Baker CA. Methylcellulose and sodium carboxymethylcellulose: An evaluation for use in paper conservation through accelerated aging. Stud Conserv. 1984;29:13.

43. Casoli A, Di Diego Z, Isca C. Cleaning painted surfaces: evaluation of leaching phenomenon induced by solvents applied for the removal of gel residues. Environ Sci Pollut Res. 2014;21:23.

44. Goldberg LA. A fresh face for Samuel Gompers: methyl cellulose poultice cleaning. J Am Inst Conserv. 1989;28:1.

45. Tserevelakis GJ, Tsagkaraki M, Siozos P, Zacharakis G. Uncovering the hidden content of layered documents by means of photoacoustic imaging Strain. 2019;55:2.

46. Tserevelakis GJ, Dal Fovo A, Melessanaki K, Fontana R, Zacharakis G. Photoacoustic signal attenuation analysis for the assessment of thin layers thickness in paintings. J Appl Phys. 2018;123:12.

47. Dal Fovo A, Tserevelakis GJ, Papanikolaou A, Zacharakis G, Fontana R. Combined photoacoustic imaging to delineate the internal structure of paintings. Opt Lett. 2019;44:4.
48. Treeby BE, Cox BT. Progress in biomedical optics and imaging. Proc SPIE, 2009;7177:717716.

49. Cherry SR, Badawi RD. Qi J. Essentials of in vivo biomedical imaging. Boca Raton: CRC Press; 2016.

50. Filippidis $\mathrm{G}$, et al. Assessment of in-depth degradation of artificially aged triterpenoid paint varnishes using nonlinear microscopy techniques. Microsc Microanal. 2015;21:510-7.

51. Oujja M, et al. Nonlinear imaging microscopy for assessing structural and photochemical modifications upon laser removal of dammar varnish on photosensitive substrates. Phys Chem Chem Phys. 2017;19:34.

52. Psilodimitrakopoulos S, Gavgiotaki E, Melessanaki K, Tsafas V, Filippidis G. Polarization Second Harmonic Generation discriminates between Fresh and Aged Starch-Based Adhesives Used in Cultural Heritage. Microscopy and Microanalysis. 2016;22:1-12.

53. Latour G, Echard J-P, Didier M, Schanne-Klein M-C. In situ 3D characterization of historical coatings and wood using multimodal nonlinear optical microscopy. Optics Express. 2012;20:24623-35.

54. Latour $\mathrm{G}$, et al. Correlative nonlinear optical microscopy and infrared nanoscopy reveals collagen degradation in altered parchments. Sci Rep. 2016:6:26344.

55. Tserevelakis GJ, Tsafas V, Melessanaki K, Zacharakis G, Filippidis G. Combined multiphoton fluorescence microscopy and photoacoustic imaging for stratigraphic analysis of paintings. Opt Lett. 2019;44:5.

56. Tserevelakis GJ, Siozos P, Papanikolaou A, Melessanaki K, Zacharakis G. Non-invasive photoacoustic detection of hidden underdrawings in paintings using air-coupled transducers. Ultrasonics. 2019;98:94-8.

57. Papadakis V, Loukaiti A, Pouli P. A spectral imaging methodology for determining on-line the optimum cleaning level of stonework. J Cult Herit. 2010;11:3.

58. Márton ZS, et al. Holographic testing of possible mechanical effects of laser cleaning on the structure of model fresco samples. NDT E International. 2014;63:53-9.

59. Fortes FJ, Cabalín LM, Laserna JJ. The potential of laser-induced breakdown spectrometry for real time monitoring the laser cleaning of archaeometallurgical objects. Spectrochim Acta Part B. 2008;63:10.

60. Tserevelakis GJ, et al. On-line photoacoustic monitoring of laser cleaning on stone: Evaluation of cleaning effectiveness and detection of potential damage to the substrate. J Cult Herit. 2019;35:108-15.

61. Papanikolaou A, et al. Development of a hybrid photoacoustic and optical monitoring system for the study of laser ablation processes upon the removal of encrustation from stonework. Opto-Elect Adv. 2020;3:190037.

62. Biqin D, Sun C, Zhang HF. Optical detection of ultrasound in photoacoustic imaging. IEEE Trans Biomed Eng. 2017;64:1.

63. Deán-Ben XL, Pang GA, Espinosa F, Razansky D. Non-contact optoacoustic imaging with focused air-coupled transducers. Appl Phys Lett. 2015;107:5.

64. Zhu Y, et al. Light emitting diodes based photoacoustic imaging and potential clinical applications. Sci Rep. 2018;8:9885.

65. Kellnberger $\mathrm{S}$, et al. Optoacoustic microscopy at multiple discrete frequencies. Light. 2018;7:109.

66. Tzoumas S, Ntziachristos V. Spectral unmixing techniques for optoacoustic imaging of tissue pathophysiology. Philosoph Trans R Soc A. 2017:375:2107.

67. Allman D, Reiter A, Bell MAL. Photoacoustic source detection and reflection artifact removal enabled by deep learning. IEEE Trans Med Imag. 2018;37:6.

68. Davoudi N, Deán-Ben XL, Razansky D. Deep learning optoacoustic tomography with sparse data. Nat Mach Intellig. 2019;1:453-60.

\section{Publisher's Note}

Springer Nature remains neutral with regard to jurisdictional claims in published maps and institutional affiliations. 